\title{
Endoftalmite bilateral por Propionibacterium acnes após facectomia com implante não simultânea
}

\author{
Bilateral Propionibacterium acnes endophthalmitis following cataract extraction with \\ non simultaneous for implant
}

Ana Luisa Höfling-Lima ${ }^{(1)}$

Ana Maria Petrilli (2)

Maria Cecília Zorat Yu ${ }^{(3)}$

Michel Eid Farah ${ }^{(4)}$

Alessandra Pinheiro Chaves ${ }^{(5)}$

\section{RESUMO}

Objetivo: Relatar um caso clínico de endoftalmite bilateral por Propionibacterium acnes no pós-operatório tardio de facectomia extracapsular não-simultânea. Relato do Caso: Paciente de 56 anos, do sexo masculino, submetido a facectomia extracapsular bilateral nãosimultânea em 1998 com intervalo de 7 meses entre uma cirurgia e outra. Quatro meses após a primeira cirurgia (olho esquerdo) desenvolveu processo inflamatório inespecífico da câmara anterior e quatro meses após a segunda cirurgia (olho direito) apresentou também reação inflamatória. Após tratamento intenso com corticosteróide tópico, peribulbar e sistêmico por quase um ano, o paciente foi submetido a paracentese de câmara anterior, punção vítrea e do espaço endocapsular; as amostras submetidas a cultura propiciaram o isolamento do $P$. acnes. Foi realizada vitrectomia e injeção de vancomicina na cavidade vítrea e na câmara anterior do olho esquerdo, que evoluiu para pthisis bulbi. O olho esquerdo submetido a vitrectomia anterior, injeção de vancomicina, explante da lente intra-ocular e remoção dos restos capsulares, evoluiu com acuidade visual corrigida de 20/70. Conclusão: A apresentação de um quadro de endoftalmite bilateral não-simultânea no pós-operatório tardio de facectomia com implante de lente intra-ocular nos alerta para a necessidade de uma completa investigação clínica e laboratorial diante de um processo inflamatório ativo após um procedimento cirúrgico, incluindo avaliação da microbiota do fundo-de-saco conjuntival para afastar a possibilidade de contaminação exógena antes da indicação do mesmo procedimento cirúrgico no olho contra lateral.

Descritores: Endoftalmite; Propionibacterium acnes; Extração de catarata.

\section{INTRODUÇÃO}

Endoftalmite caracteriza um processo inflamatório intra-ocular predominantemente centrado na cavidade vítrea e/ou câmara anterior do globo ocular, que pode ser de etiologia infeciosa ou não infecciosa ${ }^{1}$. A endoftalmite mais freqüente no pós-operatório de catarata é a de etiologia infecciosa por contaminação exógena e pode manifestar-se clinicamente no pós-operatório precoce ou tardio (30 dias ou mais após o início do tratamento) ${ }^{2}$. Em geral as fontes de contaminação são pela microbiota do fundo-de-saco conjuntival do próprio paciente ou pela utilização de soluções ou objetos contaminados ${ }^{3}$.

Os sinais e sintomas da endoftalmite variam de intensidade dependendo do agente etiológico, tempo de evolução e resistência do portador ${ }^{4}$. Nas endoftalmites de manifestação tardia os agentes etiológicos mais 
freqüentes são os fungos, bactérias de baixa virulência como o $P$. acnes e outras bactérias relacionadas a situações específicas, tais como bolha filtrante, vítreo encarcerado ou parcialmente suprimidas pelo uso de antibióticos no pósoperatório ${ }^{5}$. O Propionibacterium acnes é um microrganismo anaeróbio de crescimento lento, podendo estar presente na flora da superfície ocular ${ }^{3}$. Casos de endoftalmite no pósoperatório tardio por Propionibacterium acnes já foram previamente relatados ${ }^{6-10}$.

A ocorrência de endoftalmite bilateral por $P$. acnes no pósoperatório de cirurgias oftalmológicas não simultâneas não foi previamente descrita na literatura consultada. Ormerod et al ${ }^{6}$ avaliaram 18 casos de endoftalmite por anaeróbios onde $P$. acnes foi o agente etiológico mais comum ( $78 \%$ casos), sendo 7 casos no pós-operatório (PO) de cirurgia de catarata, 6 casos após trauma penetrante, 2 casos após transplante de córnea, 2 casos em blebites e 1 caso de endoftalmite endógena. Chien et al ${ }^{7}$ relataram um caso de endoftalmite por $P$. acnes após facectomia intracapsular com implante de lente de câmara anterior (CA), onde houve persistência do processo inflamatório por 5 anos após a cirurgia, apesar do tratamento instituído, que incluiu corticosteróide tópico e oral, antibióticos fortificados tópicos, vitrectomia via pars plana e remoção da lente intra-ocular (LIO), no entanto o controle da inflamação só foi possível após remoção do foco infeccioso, caracterizado como uma placa esbranquiçada na face posterior da córnea, associada a antibioticoterapia intra-vítrea e oral.

Manners et al ${ }^{8}$ relataram um caso de abscesso da cápsula posterior por P. acnes e S. epidermidis no PO de facectomia extracapsular, que após vitrectomia e remoção da cápsula, evoluiu com resolução completa da inflamação, sem necessidade de explante da LIO. Abrahams relatou um caso de endoftalmite por $P$. acnes com apresentação atípica caracterizada por nódulo solitário no endotélio corneano associado a uveíte granulomatosa. O diagnóstico foi feito por biópsia do nódulo ${ }^{9}$. Posenauer relatou 5 casos de infecção no pósoperatório tardio por $P$. acnes ressaltando a importância do diagnóstico diferencial entre uveíte crônica e a endoftalmite de aparecimento $\operatorname{tardio}{ }^{10}$.

\section{OBJETIVOS}

Relatar um caso de endoftalmite bilateral por Propionibacterium acnes no $\mathrm{PO}$ de facectomia extracapsular não simultânea.

\section{RELATO DE CASO}

J. S. B., 56 anos, masculino, encaminhado para nossa avaliação no PO de facectomia extracapsular com queixa de embaçamento em ambos os olhos ( $\mathrm{AO}$ ) há aproximadamente 1 ano. Relatava que 4 meses após a primeira cirurgia do $\mathrm{OE}$, teve o diagnóstico de inflamação intra-ocular que, após a admi- nistração de corticosteróide tópico, evoluiu com melhora seguida pela recorrência dos sintomas após a redução da medicação. Neste mesmo olho verificou-se a opacificação da cápsula posterior, sendo realizada a capsulotomia com YAG Laser. Sete meses após a cirurgia do $\mathrm{OE}$ foi submetido a facectomia extracapsular do OD. O procedimento cirúrgico foi feito sem intercorrências pela técnica extracapsular e após o quarto mês de cirurgia, o OD também apresentava processo inflamatório, passando a receber corticosteróide tópico agora em $\mathrm{AO}$, apesar do tratamento instituído a inflamação progrediu. $\mathrm{O}$ paciente não apresentou lesões no fundo do olho ou aumento da pressão intra-ocular, sendo portanto realizadas 2 injeções de Depomedrol ${ }^{\circledR}$ no OE com intervalo de 2 meses. Um ano após a cirurgia do primeiro olho e sete meses após a cirurgia do segundo olho o paciente foi examinado por nossa equipe e verificamos reação de CA em AO intensa, vítreo no OE e opacidades brancas endocapsulares em ambos os olhos (Figuras 1 e 2).

$\mathrm{O}$ olho esquerdo, que se apresentava com reação mais intensa foi submetido a vitrectomia posterior e biópsia vítrea, punção da CA e do saco capsular. Nas amostras analisadas obtivemos crescimento positivo do vítreo e saco capsular em

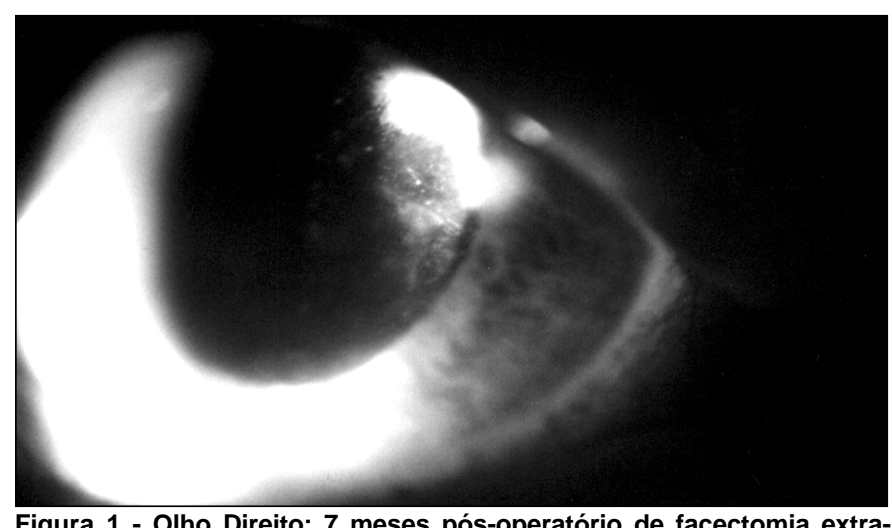

Figura 1 - Olho Direito: 7 meses pós-operatório de facectomia extracapsular com implante de lente intra-ocular com reação de câmara anterior e opacidade endocapsular

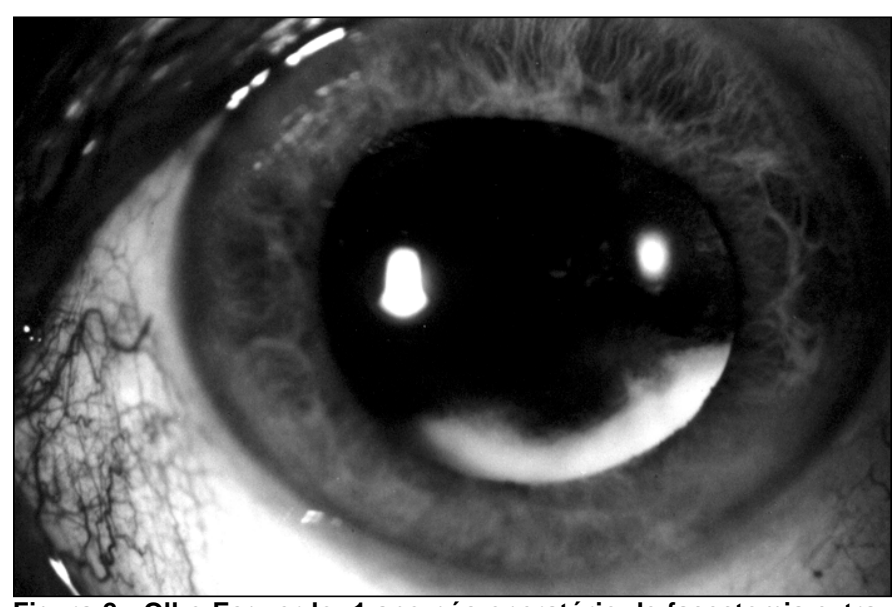

Figura 2 - Olho Esquerdo: 1 ano pós-operatório de facectomia extracapsular com implante de lente intra-ocular de câmara anterior, reação vítrea e opacidade endocapsular 
anaerobiose com colônias de cocos Gram positivos identificados como Propionibacterium acnes. Ao final do procedimento cirúrgico o paciente foi submetido a injeção intra-vítrea de vancomicina, evoluindo no pós-operatório com reação inflamatória intensa Snow Balls na retina periférica (Figura 3) e descolamento de retina (DR). Embora não fosse possível realizar uma avaliação completa da periferia da retina devido ao processo inflamatório, pelo tamanho das bolsas, havia a suspeita de DR com ruptura, foi então indicado nova vitrectomia ampla com injeção de C3F8 (Figura 4). Evoluiu no pós-operatório com intensa reação inflamatória e hipotonia intratável, mesmo com altas doses de corticosteróides e vancomicina endovenosa. As amostras colhidas no segundo procedimento do olho esquerdo do saco capsular foram também positivas.

Concomitante ao tratamento cirúrgico do $\mathrm{OE}$ houve piora da reação inflamatória do OD, sendo feita então a indicação de vitrectomia com remoção do implante intra-ocular e do remanescente do saco capsular e com injeção de vancomicina

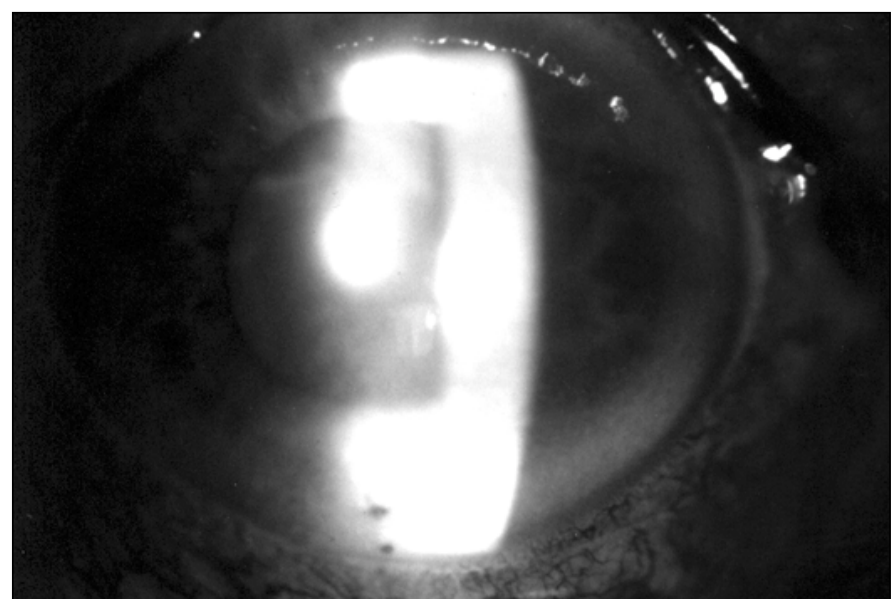

Figura 3 - Olho Esquerdo: Pós-operatório de vitrectomia com C3F8, mostrando reação inflamatória intensa

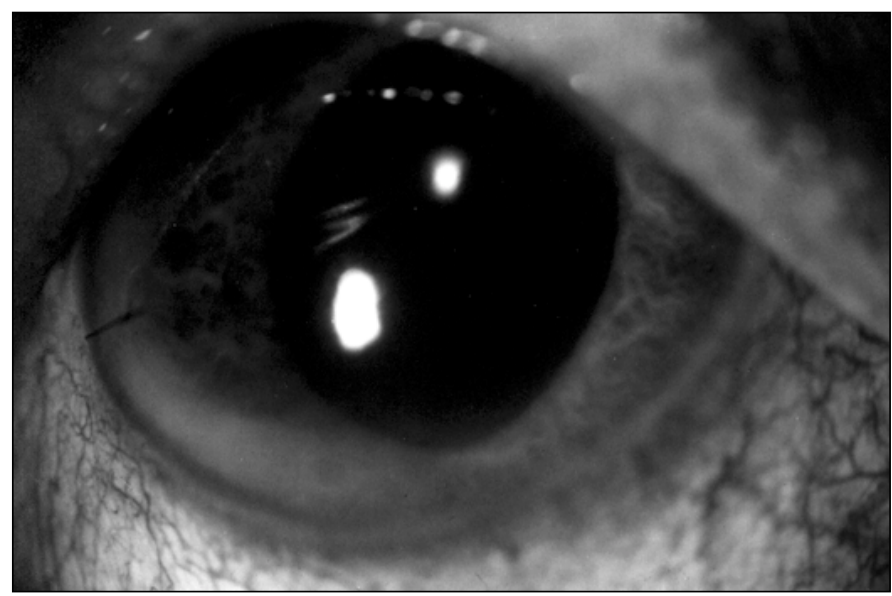

Figura 4 - Olho Direito: Pré-operatório do explante da lente intra-ocular após uma injeção de vancomicina

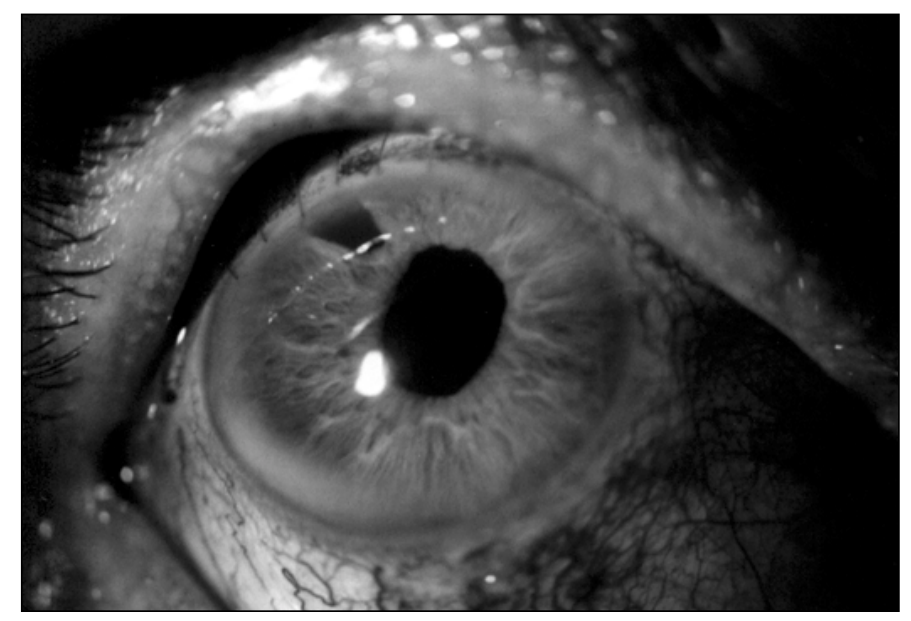

Figura 5 - Olho Direito: 1 mês pós-operatório de explante de lente intraocular sem sinais de inflamação

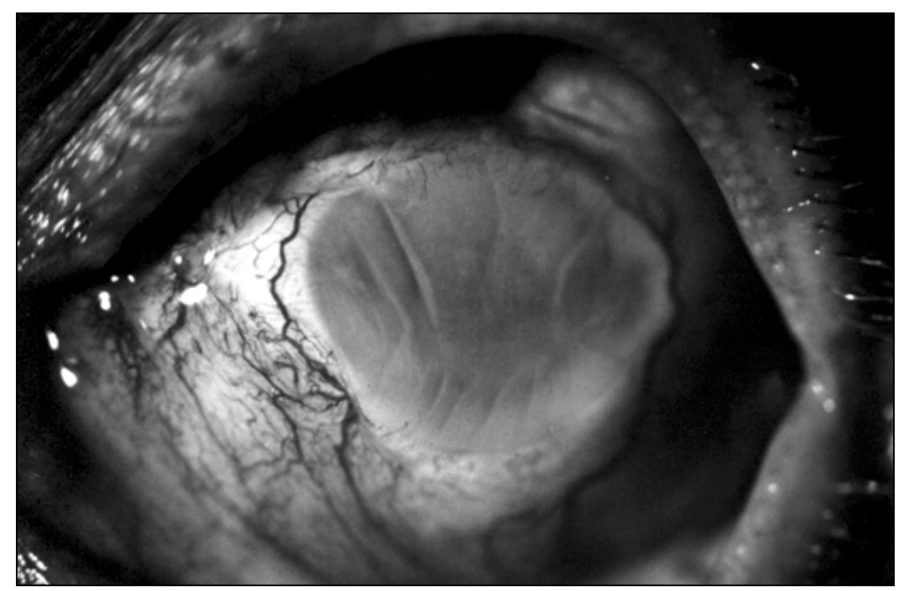

Figura 6 - Olho Esquerdo: Pthisis bulbi

intra-ocular. (Figura 5). A cultura do material obtido do saco capsular e da lente intra-ocular do OD foi positiva para Propionibacterium acnes, sendo negativa a cultura das amostras obtidas da CA e do vítreo. No pós-operatório imediato o paciente apresentava acuidade visual de movimentos de mão devido a intensa reação inflamatória. Uma segunda punção vítrea para cultura e nova injeção de vancomicina foi realizada no $4^{\circ} \mathrm{PO}$; esta segunda cultura realizada neste olho foi negativa.

Após 4 meses de evolução, o OD apresentava-se calmo, afácico e sem sinais de recidiva do processo inflamatório, o $\mathrm{OE}$ em processo de atrofia, com percepção e projeção parcial de luz (Figura 6).

\section{DISCUSSÃO}

Hall et al ${ }^{11}$ estabeleceram curvas de crescimento para $P$. acnes isolados de casos crônicos de endoftalmite pós-operatória, concluindo que o seu crescimento é mais lento que outros 
microrganismos anaeróbios, o que pode justificar o seu crescimento lento nas culturas obtidas a partir de amostras oculares, bem como ausência de resposta ao tratamento, caso a concentração de antibiótico injetado no vítreo não permaneça em níveis eficazes durante a fase crítica da replicação do organismo.

Winward et al ${ }^{12}$ propuseram substituição da LIO associada a remoção capsular completa na fase aguda da endoftalmite, justificando que a recorrência da endoftalmite por $P$. acnes é secundária a persistência de organismos viáveis sequestrados no saco capsular. Shaarawy et al ${ }^{13}$. Realizaram um estudo em 42 olhos com diagnóstico clínico e laboratorial de endoftalmite de diferentes etiologias, sendo necessário nova cultura do vítreo e uma segunda injeção intra-vítrea em 22 olhos, dos quais 12 permaneceram com culturas positivas e evoluíram com um pior prognóstico visual. Os autores concluíram que uma segunda injeção intra-vítrea de antimicrobianos deve ser considerada em algumas circunstâncias.

Endoftalmite pós-operatória por $P$. acnes é uma condição caracterizada por exacerbações e remissões, freqüentemente acompanhada por recorrências após o tratamento ${ }^{12}$, o que foi observado no caso descrito.

O aparecimento de inflamação intra-ocular no pós-operatório tardio é comumente observado em casos de endoftalmite por Propionibacterium acnes ${ }^{6-7,7-10,12-14}$, sendo que raramente existe a formação de hipópio ou abscessos. Winward sugere que a recorrência da endoftalmite por $P$. acnes é secundária à persistência de microrganismos viáveis no saco capsular ${ }^{12}, \mathrm{o}$ que deve ser favorecido pela situação de anaerobiose observada neste local. O local com melhor positividade em nossas culturas também foi o saco endocapsular.

Zambrano et al ${ }^{14}$ preconizaram que nos casos de endoftalmite a conduta inicial seria injeção intra-vítrea, seguida por antibioticoterapia específica tópica e sistêmica se necessário. Diante da ausência de resposta clínica favorável estariam indicadas então a vitrectomia via pars plana, capsulectomia e antibioticoterapia intra-vítrea. O explante da LIO e a remoção dos restos do saco capsular estariam indicados após estes procedimentos, se não houvesse melhora do quadro clínico ou nos casos mais avançados.

Neste caso, observamos que apesar da cultura do vítreo ser positiva apenas no OE, o estudo microbiológico do saco capsular foi positivo para P. acnes em AO. A melhor evolução foi observada no olho submetido a vitrectomia, com explante da LIO e remoção do saco capsular. De relevância neste caso observamos a endoftalmite bilateral por $P$. acnes após facectomia não simultânea, sendo que o olho com pior evolução foi aquele no qual o processo infeccioso teve maior duração, havia comunicação de câmara anterior com cavidade vítrea pela capsulotomia com Yag laser e teve descolamento de retina após a vitrectomia posterior, sem explante da LIO.

\section{ABSTRACT}

Purpose: To report a case of bilateral Propionibacterium acnes endophthalmitis after extracapsular cataract extraction. Case
Report: Male, 56 years old, underwent bilateral nosimultaneous extracapsular cataract extraction in 1998, followed by the second surgery 7 months after the first. Four months after each surgery, an unspecific inflammatory response was diagnosed. Almost 1 year after intense topical, peribulbar and systemic steroid treatment, he underwent anterior chamber paracentesis, vitreous and endocapsular puncture, when P. acnes was isolated. A vitrectomy was performed and vancomycin was injected into the vitreous chamber and the anterior chamber of his left eye, whose evolution was pthisis bulbi his right eye was submitted to vitrectomy, vancomycin injection and intraocular lens explantation, recovering a best corrected visual acuity of 20/70. Conclusion: Late bilateral non simultaneous endophthalmitis presentation after cataract extracapsular extraction with intraocular lens implant leads us to a complete clinical and laboratorial investigation facing an active inflammatory response after the first surgery, including microbiological conjunctival cul-de-sac evaluation in order to rule out the possibility of exogenous contamination before the fellow eye can undergo the same surgical procedure.

Keywords: Endophthalmitis; Propionibacterium acnes; Cataract extraction.

\section{REFERÊNCIAS}

1. Chaib AR, Freitas D, Scarpi MJ, Guidugli T. Pesquisa laboratorial em endoftalmite. Arq Bras Oftalmol 1997;60:250-7.

2. Moreira Jr. CA, Schneider JR, Portella E. Endoftalmites. Rev Bras Oftalmol 1997;56:627-30.

3. Tervo T, Ljungberg P, Kautiainen T, Puska P, Lehto I, Raivio I, Järvinen E, Kuusela P, Tarkkanen A. Prospective evaluation of external ocular microbial growth and aqueous humor contamination during cataract surgery. J Cataract Refract Surg 1999;25:65-71.

4. Molinari LC, Peyman GA. Guia prático para o manejo da endoftalmite. Rev Bras Oftalmol 1996;55:647-58.

5. Cullom Jr. RD, Chang B. The Wills Eye Manual, $2^{\text {nd }}$ ed Philadelphia: JB Lippincott; 1993. p. 376-86.

6. Ormerod LD, Paton BG, Haaf J, Topping TM, Baker AS. Anaerobic bacterial endophtalmitis. Ophthalmology 1987;94:799-808.

7. Chien AM, Raber IM, Fischer DH, Eagle Jr. RC, Naidoff MA. Propionibacterium acnes endophthalmitis after intracapsular cataract extraction. Ophthalmology 1992;99:487-90.

8. Manners RM, Canning CR. Posterior lens capsule abscess due to Propionibacterium acnes and Staphylococcus epidermidis following extracapsular cataract extraction. Br J Ophthalmol 1991;75:710-2.

9. Abrahams IW. Propionibacterium acnes endophthalmitis: an unusual manner of presentation. J Cataract Refract Surg 1989;15:698-701.

10. Posenauer B, Funk J. Chronic postoperative endophthalmitis caused by Propionibacterium acnes. Eur J Ophthalmol 1992;2:94-7.

11. Hall GS, Pratt RK, Meisler DM, Washington JA, Roussel TJ, Miller D. Growth curve for Propionibacterium acnes. Curr Eye Res 1994;13:465-6.

12. Winward KE, Pflugfelder SC, Flynn Jr. HW, Roussel TJ, Davis JL. Postoperative Propionibacterium endophthalmitis. Treatment strategies and long-term results. Ophthalmology 1993;100:447-51.

13. Shaarawy A, Grand MG; Meredith TA, Ibanez HE. Persistent endophthalmitis after intravitreal antimicrobial therapy. Ophthalmology 1995;102:382-7.

14. Zambrano W, Flynn Jr. HW, Pflugfelder SC, Roussel TJ, Culbertson WW, Holland S, Miller D. Management options for Propionibacterium acnes endophthalmitis. Ophthalmology 1989;96:1100-5. 